\title{
El enfoque por competencias: un acercamiento a la práctica docente
}

\author{
The competency approach: \\ an approach to teaching practice
}

DOI: http://dx.doi.org/10.17981/cultedusoc.12.2.2021.02

Recibido: 19 de septiembre de 2020 Aceptado: 14 de diciembre de 2020 Publicado: 1 de julio de 2021.

Isabel Guzman-Ibarra (1)

Universidad Autónoma de Chihuahua. Chihuahua (México)

iguzman@uach.mx

Rigoberto Marín-Uribe

Universidad Autónoma de Chihuahua. Chihuahua (México)

rmarin@uach.mx

Cintia Lizzette Ortega-Alderete (i)

Universidad Autónoma de Chihuahua. Chihuahua (México)

iguzman@uach.mx

Para citar este artículo:

Guzman-Ibarra, I., Marín-Uribe, R. y Ortega-Aldete, C. (2021). El enfoque por competencias: un acercamiento a la práctica docente. Cultura, Educación y Sociedad, 12(2), 27-48. DOI: http://dx.doi.org/10.17981/cultedusoc.12.2.2021.02

\section{Resumen}

Este artículo comunica resultados de un estudio cualitativo realizado en una institución de educación superior de la ciudad de Chihuahua (Chihuahua, México), con 12 profesores. Describe viviencias en tres categorías de práctica docente por competencias: planeación, implementación y evaluación. La investigación fue de corte etnográfico empleando como técnicas el análisis documental, la observación y la videograbación. El análisis de datos se realizó mediante una matriz de análisis, considerando la práctica docente como unidad de análisis y a los momentos de planeación didáctica, intervención en el aula y evaluación de competencias, como categorías de análisis. Los resultados muestran la comprensión de los profesores acerca de: enseñanza, aprendizaje y evaluación en su práctica cotidiana. Se categorizaron tres grupos: el primero, ha interiorizado el enfoque por competencias; el segundo, evidencia avances en planeación didáctica y pocos en la evaluación de competencias; y un tercer grupo, continua su práctica tradicional. Se concluye que estos profesores, se encuentran en una etapa de transición de prácticas docentes tradicionales hacia el modelo por competencias, lo cual impacta en una escasa identidad y compenetración con el enfoque.

Palabras clave: Enfoque por competencias; evaluación de competencias; análisis de práctica educativa

\section{Abstract}

This article communicates the results of a qualitative study carried out in a higher education institution in the city of Chihuahua (Chihuahua, Mexico), with 12 professors. It describes experiences in three categories of teaching practice by competencies: planning, implementation and evaluation. The research was ethnographic in nature that used documentary analysis, observation and video recording as techniques. The data analysis was carried out using an analysis matrix, considering teaching practice as the unit of analysis and the moments of didactic planning, classroom intervention and evaluation of competencies, as categories of analysis. The results show the teachers' understanding of: teaching, learning and assessment in their daily practice. Three groups were categorized: the first, has internalized the approach by competencies; the second shows progress in didactic planning and few in the evaluation of competences; and a third group continues their traditional practice. It is concluded that these teachers are in a stage of transition from traditional teaching practices towards the competency model, which impacts on a scarce identity and rapport with the approach.

Keyworks: Approach by competences; evaluation of competences; analysis of educational practice 


\section{INTRODUCCIÓN}

Los modelos por competencias en México han sido implementados en todos los niveles educativos, a partir de interpretaciones, recursos, acciones y estrategias muy diversas. Después de más de veinte años de su instrumentación es menester investigar sobre las experiencias vividas al respecto por profesores y estudiantes. Para su puesta en práctica las instituciones se vieron precisadas a migrar sus programas, a la par de impulsar acciones a fin de responder a esta nueva política educativa (Robles-Haros y Estévez-Nenninger, 2016). Este artículo tiene como propósito comunicar los resultados de un estudio de corte etnográfico, realizado en una institución de educación superior politécnica de la ciudad de Chihuahua (Chihuahua, México), centrado en el trabajo educativo cotidiano de un grupo de profesores quienes se desempeñan en el campo de la administración. Se analizaron las concepciones de los profesores respecto de algunos constructos pedagógicos relacionados con su praxis y con la forma de implementarlos en el aula, así como su articulación con los sustentos del enfoque por competencias.

La institución, con doce años de existencia y operación del modelo educativo por competencias, está orientada a la formación tecnológica, la formación inicial de los profesores corresponde a diversas disciplinas profesionales, en ese sentido, la docencia representa una segunda profesión; en esta doble identidad, la formación inicial ocupa un papel proponderante (Guzmán y Marín, 2011). De ahí la motivación por acercarse a investigar sus procesos de docencia, las concepciones construidas y operacionalizadas en las aulas, las estrategias puestas en práctica en la concresión del modelo, así como los avances, estancamientos, encuentros y desencuentros en la interpretación y aplicación del mismo, que si bien está presente en las instituciones educativas, los espacios para la discusión y análisis de los principales referentes teóricos y prácticos, han sido escasos en esta institución.

El interés por innovar en los procesos de formación y pugnar por un cambio, es preocupación constante de las comunidades educativas; en nuestro país se han dado últimamente una serie de reformas orientadas al desarrollo social, económico y educativo, como una apuesta para contribuir al mejoramiento de la calidad de vida. En estas reformas el papel del profesor es altamente significativo, ya que gran parte de las reformas descansan en él y, paradójicamente, se le demanda mayor formación para asegurar un verdadero cambio en la educación. En esta articulación de sociedad-economía-educación, la labor de los docentes es trascendente, no solo en el discurso que al respecto deba difundirse, sino porque en sus manos queda la estrategia y la posibilidad de habilitar a los futuros profesionistas encargados de impulsar el desarrollo socioeconómico a partir de la educación.

La universidad bajo estudio, al igual que la mayoría de las instituciones mexicanas de educación superior, se vieron precisadas a adoptar el enfoque basado en competencias, (Cifuentes, Cortés, Garzón y González, 2020) tendiente a desarrollar competencias para la identificación y solución de los problemas de la realidad en el entorno de los estudiantes, buscar la incidencia de las prácticas pedagógicas en el aprendizaje e implementar estrategias para el desarrollo de las competencias en el marco del modelo educativo de la institución. En nuestra institución el modelo por competencias buscó: 
[...] responder a las necesidades sociales de formar profesionistas de manera integral, dotándolos de las competencias necesarias para integrarse a cualquier ambiente de trabajo, se busca que los alumnos adquieran capacidades generales para aprender y actualizarse; para identificar, plantear y resolver problemas; se pretende favorece el aprendizaje a través de situaciones reales de trabajo (Universidad Politécnica de Chihuahua-UPCH, 2009).

En esta institución, el proceso para la implementación del modelo competencial implicó un proceso de formación de profesores, considerado como limitado en tiempo, estrategias y número de participantes; dedicando un importante tiempo al cabildeo y negociación, De esta forma, la capacitación docente fue diluyéndose entre los profesores de semestres iniciales, con quienes se iniciaba la operación del modelo, ellos asimilaron los principios básicos del modelo, mientras que los docentes que atendían grupos de semestres finales no requirieron capacitarse, por no verse precisados a implementar cambios en su práctica.

Los procesos de capacitación contemplaron el análisis de los fundamentos teóricoprácticos del modelo. Asimismo, dado que el enfoque basado en competencias requiere de cambios en la práctica, se estableció el compromiso institucional de acompañamiento a lo largo del trayecto de su implementación. De esta forma, en ese devenir, los niveles de intranquilidad e inercia inicial fueron disminuyendo y el enfoque por competencias pasó, de ser desconocido por los profesores, a un proceso educativo que terminó por instalarse.

El éxito de una reforma, y en este caso, del enfoque basado en competencias, estuvo supeditado en gran medida a la interiorización de sus principios y fundamentos, así como en la forma en que estos se implementaran en el aula, atendiendo a lo expresado en el discurso en cuanto a que

La educación en la actualidad tiene como misión esencial la formación de profesionales altamente capacitados que actúen como ciudadanos responsables, competentes y comprometidos con el desarrollo social, ello implica que el proceso de formación de los docentes de cualquier nivel educativo estén llenos de conocimientos y habilidades integrales que permita cumplir con las funciones que requiere su profesión, por este motivo la capacitación no es una herramienta más para cumplir con las necesidades estudiantiles es una obligación que debe ser practicada en cualquier institución educativa (Rodríguez, 2016, párr. 15).

Así, el enfoque por competencias, permanece en nuestras instituciones, con los cambios y adecuaciones necesarias o acudiendo a las propias lógicas y creencias que los profesores han realizado para el desarrollo de su práctica cotidiana (Londoño y Luján, 2020). Las competencias son consideradas como un principio organizador del currículo, encontrando en la mayoría de las instituciones que han implementado este enfoque una clasificación de las competencias en: básicas o instrumentales, que deban poseer todos los estudiantes de la universidad; profesionales, que identifican el desarrollo de un campo profesional; y las específicas, que son muy particulares de un área o disciplina. Atendiendo a esta clasificación, los profesores se familiarizan con los grandes desempeños hacia los cuales se orientan los contenidos de la carrera que imparten. Del mismo 
modo, si se tiene claridad sobre dichos desempeños, se establece el perfil profesional basado en los tres tipos de competencias mencionados, lo cual permite transversalizar los contenidos propios de cada asignatura.

Tomando como base los principios en los cuales descansa el enfoque por competencias, este estudio se orientó al análisis de tres momentos de la práctica docente por competencias: planeación didáctica, intervención en el aula y evaluación de competencias. Para tal propósito, se consideró importante trabajar en torno a los elementos de la noción de competencias, a fin de llevarlos al campo de prácticas educativas por competencias, con esto se buscó que los tres momentos mencionados, estuvieran conformados y se desarrollaran por el sentido del concepto que las anima. De esta manera, se pretendió partir de un concepto de competencias que unificara estos tres momentos y, en su ejercicio, les diera congruencia.

Así, más que una definición, se tomó un conjunto de elementos comunes en la discusión sobre la noción de competencias, concibiéndose de esta forma como: la capacidad para movilizar un conjunto de recursos cognitivos (saberes, capacidades, microcompetencias, informaciones, valores, actitudes, esquemas de percepción, de evaluación y de razonamiento) [...] entre otros, que una persona, moviliza para resolver situaciones problema complejas) (Guzmán y Marín, 2011, p. 154).

En consecuencia, la planeación didáctica deberá ser entendida como un momento que parta de situaciones problemas, de las cuales se desprendan aquellas actividades de aprendizaje que lleven a los estudiantes a enfrentarlas para movilizar sus recursos cognitivos y en ese proceso desarrollar las competencias que se planean. Esta planeación, llevada al aula para su realización, implica una práctica docente orientada al desarrollo de competencias basada en situaciones complejas (Brosseau, 2007; Roegiers, 2010), la búsqueda de solución a las mismas, conduce a la adquisición de las competencias que han de concretarse en evidencias de desempeño. Es necesario tener claro cómo aprende el alumno, ya que la tarea del profesor será la de apoyar estas formas de aprendizaje y modalidades en que el estudiante evidencia sus aprendizajes en desempeños, esto conduce a la evaluación de competencias.

Congruentes con lo anterior la evaluación estará articulada con la evidencia preestablecida y negociada desde la misma planeación, así como la forma de concretar esta evidencia. La evaluación basada en desempeños, no debera ser más una evaluación de contenidos, sino de los desempeños mostrados por los estudiantes al resolver las situaciones a las que se enfrentan (Roegiers, 2016), en esta concepción de evaluación se observa que convergen los elementos explicitados en el concepto del que partimos en donde las competencias comportan "la capacidad para movilizar diversos recursos cognitivos que permiten hacer frente a situaciones complejas en un contexto determinado de forma interrelacionada y pertinente" (Guzmán, Marín e Inciarte, 2014, p. 10). La evaluación entonces, en este enfoque, representa la oportunidad para transcender prácticas rutinarias y el propio carácter de la evaluación, pues la evaluación de competencias implica situar a los estudiantes ante situaciones o familia de situaciones complejas con la intención de que pueda resolverla movilizando sus recursos cognitivos, estas situaciones deberán ser reales lo que le imprime al proceso un sentido de evaluación auténtica (Moria, Refnaldi \& Zaim, 2017). 
Sin desconocer las condiciones en las cuales ha sido implementada, en nuestro contexto la educación basada en competencias se ha caracterizado por altibajos, por un lado, se nos presentó como una apuesta innovadora, por otro, ha sido criticada por la falta de debate sobre sus principios, origen y su implementación en el ámbito educativo. Observamos que cada nivel educativo que ha adoptado el modelo por competencias ha desarrollando las estrategias y acciones pertinentes para su implementación, mostrando un avance gradual, como graduales fueron las reformas que dieron pie a cada implementación (Marín, Guzmán, Inciarte y Araya, 2015). La realidad vivenciada en nuestra institución, da cuenta de algunas imprecisiones, capacitaciones intermitentes, información poco explícita, por lo que los procesos de capacitación y operacionalización estuvieron cargados de dudas o desconocimiento, dificultando su valoración o evaluación en términos de los propósitos de la reforma.

Se estima que el debate sobre los aspectos sustantivos del modelo no estuvo ni ha estado presente en los espacios de la academia, en donde se aprecia que el propio concepto de competencias no ha sido reflexionado, cuando debería ser parte de las interacciones en los colectivos académicos para ser asumido por los profesores, en quienes finalmente recae la responsabilidad de traducirlo en acciones en el aula.

El enfoque por competencias requiere del análisis de los profesores a fin de orientar sus prácticas para hacerlas congruentes con los fundamentos del enfoque, reconociendo que la noción de competencia no proviene de un solo paradigma, ni de una sola tradición psicopedagógica, el uso y aplicación está dada con base en las necesidades y orientaciones de cada autor.

Con base en lo expuesto, el estudio realizado pretendió analizar la distancia que media entre los sustentos teóricos que fundamentan el modelo por competencias y la realidad que están viviendo los profesores en el ejercicio cotidiano de su práctica docente, al valorar que los docentes tienen percepciones diferentes del enfoque.

\section{MÉTODOLOGIA}

La investigación realizada se fundamenta en un enfoque cualitativo de corte etnográfico, donde se reconoce la intersubjetividad y los diversos símbolos y significados sociales compartidos, esto representó una alternativa para adentrarse en la comprensión de las situaciones a las cuales se enfrentan los profesores en su esfuerzo por desarrollar su práctica docente apegándose a un modelo educativo para ellos desconocido (JiménezDomínguez, 2000).

Más allá de la obtención de datos "duros" sobre los resultados presentados por los profesores o la cuantificación de sus mejores programas, desempeños o conductas, asumimos la postura cualitativa a fin de posibilitar la incorporación al análisis de las diversas miradas y perspectivas y ayudar a comprender y redimensionar la práctica educativa de los docentes y, con ello, aportar nuevos datos, incorporar su sentir-pensar y analizar las estrategias seguidas para auto-capacitarse, organizarse y comprometerse entre sí, para llevar al aula, un modelo que requieren hacer propio para darle el sentido y significado necesario para poder avanzar. 


\section{Perspectiva epistemológica del estudio}

El paradigma asumido en esta investigación se ubica en el enfoque interpretativo el cual es estudiado de manera holística, naturalista y fenomenológica. Es importante señalar que la fenomenología como visión filosófica tuvo una influencia importante en la investigación interpretativa del siglo pasado; siendo esta escuela de pensamiento la que describe el sentido de las experiencias vividas desde la perspectiva de los sujetos participantes quienes evidencian significados implícitos, este tipo de investigación interpretativo pretende analizar e interpretar en el contexto socioeducativo las relaciones y prácticas en los escenarios socioeducativos donde se desarrollan. Actualmente el discurso en torno a la fundamentación epistemológica de las ciencias sociales parece estar ausente, sin embargo, los fenomenólogos están de acuerdo y consideran la reflexividad epistemológica de su método, apoyando la idea de una articulación sistemática "del análisis fenomenológico del mundo de la vida con la sociología empírica ha demostrado ser fructuosa. Sin embargo, su relación no debe percibirse como unilateral sino como recíproca: la protosociología fenomenológica y la sociología deben corregirse mutuamente" (Eberle, 2019, p. 150).

\section{Procedimiento metodológico}

Los momentos más importantes del proceso metodológico seguido en esta investigación se esquematizan en la Figura 1 y se describen a continuación:

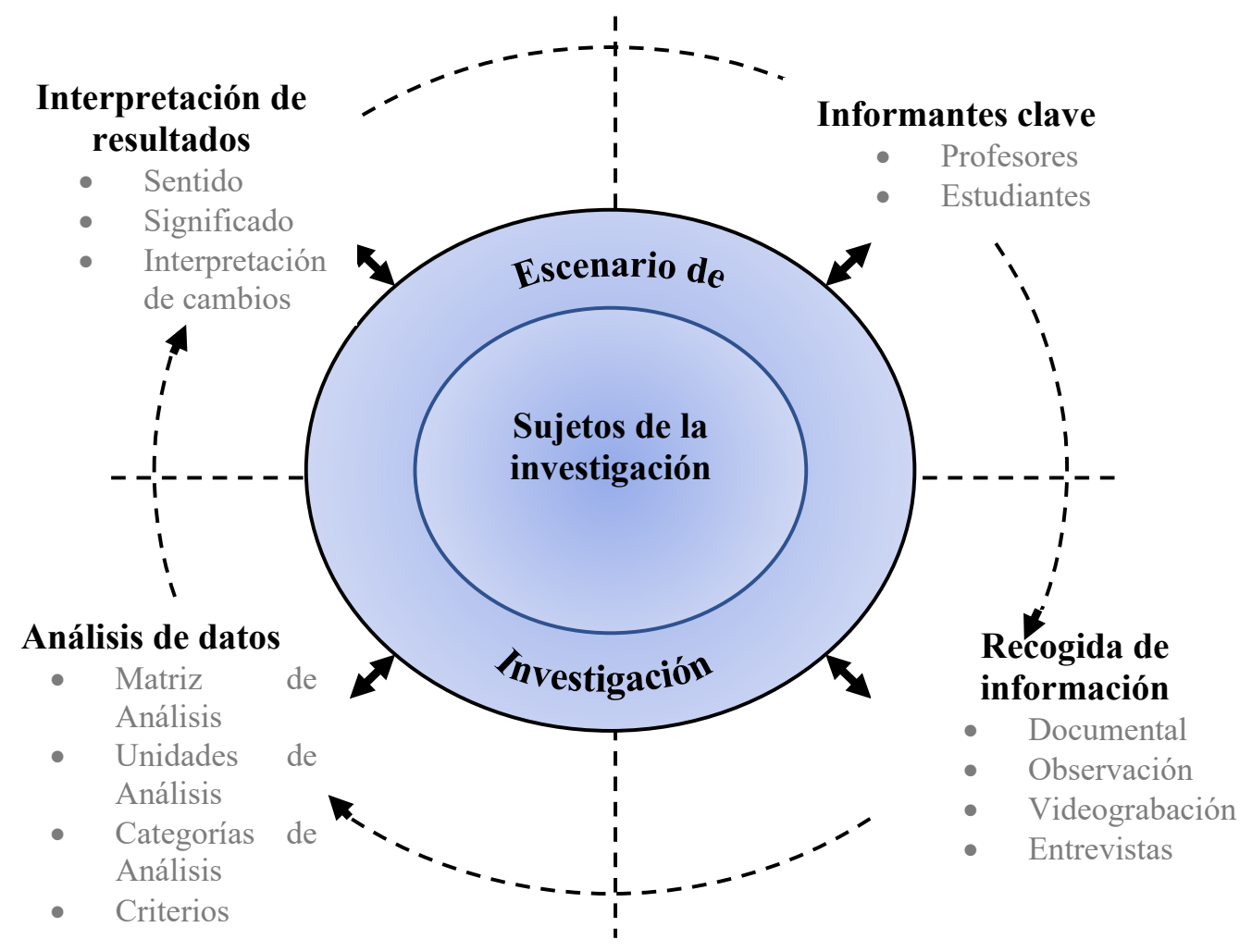

Figura 1. Procedimiento metodológico Fuente: Autores. 


\section{Escenario de la investigación}

En el diseño etnográfico, los espacios educativos representan el escenario de la investigación donde se sintetizan complicados y entrelazados procesos sociales, lo cual permite redimensionarlos desde la escuela, como los espacios cotidianos donde suceden los hechos relativos al quehacer educativo y los sujetos se manifiestan, piensan, actúan, se relacionan e interactúan, construyendo prácticas específicas y diferenciales en función de la información recabada de los participantes, entendiendo que:

[...] una de las primeras demandas del enfoque etnográfico y del investigador educativo es que no se asiste al campo para confirmar hipótesis o ideas que ya se creían saber, nociones previas alimentadas por el sentido común, sino que se entra al campo con la intención de interrogar cómo ocurren los procesos, las prácticas, la vida cotidiana en el espacio, que pudieran ser completamente ajenos o curiosamente familiares (Soto y Nieves, 2018, p. 170).

La investigación tuvo como escenario una institución pública de nivel superior del norte de México. Su oferta académica la componen cuatro licenciaturas una en el área de administración y tres en el campo de las ingenierías: Tecnología Ambiental, Mecánica Automotriz, y Aeronáutica. En este estudio se trabajó en la licenciatura en Administración.

\section{Sujetos de la investigación}

Participó el personal que labora en el área de docencia que suma un total de 23 docentes y 123 estudiantes. De este total se trabajó con una muestra significativa, que integró como informantes clave a 12 profesores que imparten las asignaturas teóricas y teórico-prácticas de la carrera de administración en los semestres iniciales y finales, así como a 10 estudiantes que cursaban esa carrera. Los informantes clave fueron seleccionados atendiendo al principio de pertinencia y significatividad de sus historias profesionales y narrativas de eventos que como protagonistas reconstruyen; los criterios de inclusión de los informantes clave tomaron como base su participación activa en los procesos educativos bajo estudio, su nivel de compromiso, responsablidad, experiencia y su interés por participar en la investigación (Chavarría-Zambrano y Camacho, 2018).

\section{Recogida de información}

Las técnicas e instrumentos que se emplearon en la recolección de la información fueron: a) la entrevista etnográfica, esta técnica facilitó la recogida de datos a partir de preguntas sobre el objeto de estudio, a través de las cuales los sujetos compartieron mediante una relación interpersonal su experiencia (opiniones, percepciones, actitudes, creencias, sentires...) sobre la temática bajo estudio, se buscó mantener una comunicación horizontal entre investigadores y sujetos investigados, las entrevistas se realizaron a 10 profesores y 10 estudiantes; b) el análisis documental, que consistió en la revisión de las planeaciones didácticas de los 12 profesores que conformaron la muestra; c) la observación etnográfica, la cual se realizó a dos profesores: uno impartía "Introducción a la Administración" (materia teórica), el otro, "Administración Financiera" (asignatura teórico-práctica), en el cuatrimestre agosto-noviembre de 2018; y d) la videograbación, esta herramienta de apoyo 
a las observaciones permitió que se levantaron en total 24 registros videograbados de la práctica observada, 12 en cada una de las materias impartidas por los dos docentes que integran la muestra.

\section{Análisis de información}

Para el análisis de la información recabada en las entrevistas, el análisis documental y observaciones se diseñó una matriz de análisis, la cual fue aplicada al estudio de la práctica docente tomada como la unidad de análisis para describir el espacio de la práctica educativa, sus momentos y las interacciones que se establecen entre profesores y estudiantes, fue concebida como el espacio perceptivo que orientó la recogida y el análisis de los datos.

La matriz de análisis fue construida a partir de tres grandes categorías: 1) Planeación didáctica; 2) intervención en el aula, y 3) evaluación de competencias. Como se observa las categorías representan los tres momentos en que se divide la práctica docente, esto condujo a identificar las regularidades, la recurrencia de eventos, los temas relevantes, así como la reducción de los datos y su clasificación. Estas categorías de análisis, con sus referentes básicos del enfoque por competencias, llevaron a la caracterización de la práctica docente a partir de criterios que facilitaron la interpretación y obtención de un panorama de la situación académica del proceso, asimismo, de la integración en grupos de los profesores en una tipología conformada con base en los resultados del estudio etnográfico.

\section{Interpretación de resultados}

Con el apoyó de la matriz de análisis se siguió la lógica de los estudios cualitativos en la interpretación de los resultados. Esta estrategia permitió: a) Un acercamiento a la información de los participantes sobre sus ideas, creencias, conocimientos, áreas de formación, incluso generó espacios de encuentro para recabar la historia, la ubicación, los niveles socioeconómicos, educativo, político y cultural de los profesores participantes; b) interpretar y describir, desde una óptica naturalista, lo que los docentes desde y en su contexto realizan en su cotidianeidad; c) interpretar el sentido y significado que los profesores dan a su práctica educativa; con este énfasis accedimos a la compresión de las implicaciones del proceso, desde la capacitación, hasta la implementación, bajo circunstancias concretas; y d) la recuperación e integración de testimonios, como datos empíricos analizados a la luz de las categorías y criterios de análisis, con lo que se logró clasificar los hallazgos, su triangulación, la reflexión teórica y la conceptualización de los investigadores.

\section{Resultados}

Los resultados referidos a la unidad de análisis "Práctica docente por competencias" se presentan a partir de tres categorías de análisis: la planeación didáctica es la categoría que, desde la fase de elaboración, diseña ambientes de aprendizaje y abre espacios para la negociación y acuerdos entre el profesor y el grupo; la categoría de intervención en el aula, enfatiza en la relación educativa dada en los procesos de enseñanza-aprendizaje 
y rescata lo referente al aspecto conceptual y a la movilización de los diversos recursos cognitivos, requeridos para resolver situaciones complejas de la vida profesional y personal que enfrentan los estudiantes, y la categoría de evaluación de competencias, que evidencia los avances y los logros alcanzados, así como la retroalimentación del proceso educativo que conduzca a la reflexión sobre las mejoras a incorporar a una práctica docente renovada.

\section{Planeación didáctica}

Esta categoría determina la directriz y el sentido a seguir por el docente para lograr transformar los saberes de los alumnos, tanto en conocimientos, como en habilidades, valores y actitudes, se debe tener en cuenta que es una guía que permite flexibilizar acciones, pero sin perder de vista el objetivo curricular (Morales, 2018). Todo inicia con la planeación y es en función de ella que se abre el panorama para avisorar la brecha que existe entre la planeación y la evaluación.

La planeación, desde la lógica de la práctica reflexiva, "nos ofrece un nuevo significado: 'reflexión sobre la acción' y ‘reflexión en la acción', como lo expresa Schön, dando a entender dos elementos constitutivos de la Práctica Reflexiva" (Domingo, 2021, p. 10), la reflexión sobre la acción, representa el análisis de la evidencia de la experiencia vivida, la recuperación de los productos de un ciclo que se ha cerrado. En ese sentido, cada momento de planeación da pie para la incorporación de cambios y transformaciones a partir de lo vivido en semestres anteriores.

En la Tabla 1 se presenta el análisis e interpretación de testimonios que dan cuenta de algunos criterios de la categoría de planeación didáctica de profesores cuya práctica docente atiende materias teóricas.

TABLA 1.

Criterios de planeación didáctica en materias teóricas.

\begin{tabular}{|c|c|c|}
\hline \multirow[t]{2}{*}{ Categoría } & Criterios & Testimonios \\
\hline & $\begin{array}{l}\text { Conocimiento } \\
\text { previo. }\end{array}$ & $\begin{array}{l}\text { A partir del tema sobre los antecedentes históricos de la administración, se } \\
\text { incorporan las principales aportaciones al respecto [...]. }\end{array}$ \\
\hline $\begin{array}{l}\text { Planeación } \\
\text { didáctica }\end{array}$ & $\begin{array}{l}\text { Intervención } \\
\text { en el aula. }\end{array}$ & $\begin{array}{l}\text { [...] En esta unidad analizaremos algunos elementos de la administración } \\
\text { donde tenemos que cubrir algunos temas, revisen el material que vamos a } \\
\text { utilizar que son los textos con el que vamos a trabajar ustedes y yo, los temas } \\
\text { son los antecedentes históricos de la administración, les traje el instrumento } \\
\text { ya impreso con el que vamos a iniciar la clase, con estas copias perfectamente } \\
\text { autorizadas, donde he planeado terminar estos temas este día. }\end{array}$ \\
\hline
\end{tabular}

Fuente: Autores.

En la Tabla 1 se contrastan dos criterios, uno, corresponde a la fase de planeación como documento, el otro, a la aplicación de este criterio en el momento de la intervención áulica. El primer criterio está referido al conocimiento previo, es entendido como la información que los estudiantes tienen en su memoria, producto de experiencias pasadas y que habrán de recuperar en el proceso de aprendizaje de nuevos contenidos y en el desarrollo de competencias. 
Este concepto, propio de la teoría de aprendizaje significativo, es un aporte de la psicología cognitiva, se emplea en la pedagogía como un apoyo importante en el proceso de enseñanza-aprendizaje, considerando que, los estudiantes, al contar con estos conocimientos previos se le facilita la adquisición de nuevos aprendizajes. En ello radica la importancia de su visualización desde el momento de la planeación, pues es en este proceso que "se identifican los conocimientos previos que los alumnos ya disponen y que les ayudarán a construir los nuevos aprendizajes que se propondrán en el problema" (Vargas y Romero, 2018, p. 255), al respecto, se encontró en la revisión documental de las planeaciones didácticas de los docentes, que éstos sí consideran la necesidad de incorporar la concepción de conocimientos previos, incluyendo temas en sus planeaciones que aporten al aprendizaje de conocimientos previos por parte de los estudiantes.

El segundo criterio, denota la aplicación del primer criterio, cuando el docente promueve la compresión de lectura como una estrategia del proceso de activación de conocimientos previos. En este caso se encauza a los estudiantes, para que de forma independiente, empleen sus conocimientos y habilidades adquiridas con antelación, para tal fin, utiliza como recursos de apoyo, documentos cuyo análisis implica una interpretación, la cual depende del conocimiento previo al ejercicio de la lectura. En este momento de intervención áulica se acude a esta estrategia conscientes de que "la activación de los conocimientos previos y experiencias del estudiante generan una estructura cognitiva, lo cual facilitará el proceso de aprendizaje significativo de un nuevo tema de estudio" (Penagos, 2015, p. 114).

En el análisis de estos dos criterios se observa el trabajo de planeación didáctica y su aplicación en un evento de intervención en el aula, como parte del proceso de desarrollo de competencia en los estudiantes, asimismo, se aprecia cómo este recurso didáctico proporciona al docente pistas sobre cuáles son las temáticas que deben ser resaltadas para ser activadas de nueva cuenta en el aula (Palma, 2017).

En contraposición con estos testimonios, en la Tabla 2 se presentan los desarrollados en la planeación didáctica de profesores que atienden materias prácticas.

TABLA 2.

Criterios de planeación didáctica en materias prácticas.

\begin{tabular}{lll}
\hline Categoría & \multicolumn{1}{c}{ Criterios } & \multicolumn{1}{c}{ Testimonios } \\
\cline { 2 - 3 } & $\begin{array}{l}\text { Estrategia } \\
\text { didáctica }\end{array}$ & $\begin{array}{l}\text { ¿Cuál será la mejor estrategia para diseñar un flujo efectivo y qué elementos } \\
\text { tendremos que incorporar? }\end{array}$ \\
$\begin{array}{l}\text { Planeación } \\
\text { didáctica }\end{array}$ & $\begin{array}{l}\text { Relación } \\
\text { educativa profesora llega al salón saludando dice, buenos días, empezamos la clase, }\end{array}$ & $\begin{array}{l}\text { vamos a comenzar a realizar un flujo efectivo, se planea hacer un estado de } \\
\text { resultados en el programa de Excel [...] La profesora bromea con los estudiantes, } \\
\text { les dice que hay alumnos que no hablan, pero tampoco trabajan, se despide la } \\
\text { maestra: nos vemos la próxima clase, que es mañana, me traen el producto [...]. }\end{array}$ \\
\hline
\end{tabular}

Fuente: Autores.

Como en la situación anterior, en esta tabla se describen dos criterios correspondientes a la planeación didáctica, en este caso, de profesores que atienden materias prácticas. El primer criterio atiende a la planeación como documento y el segundo, la aplicación de la planeación en la intervención en el aula. 
Respecto del primer criterio referido a las estrategias didácticas, el docente al iniciar su planeación parte de cuestionarse sobre cuáles serán las estrategias más adecuadas para la enseñanza del tema de "flujo efectivo" dentro de su materia práctica, esta pregunta lo lleva a reflexionar sobre cuestiones "siempre necesarias: ¿cómo aprender a enseñar lo que se enseña aprendiendo? ¿Qué elementos deben constituir un espacio para posibilitar el aprendizaje?" (Celis, Parada y Ríos, 2020, p. 8). Al referirnos al desarrollo de prácticas docentes desde el enfoque de competencias, la planeación didáctica adquiere en nuestro análisis un papel preponderante, pues ello implica la realización de procesos de búsqueda y de creatividad para diseñar ambientes de aprendizaje y estrategias didácticas pertinentes para el desarrollo de competencias en los estudiantes a fin de que sean copartícipes de su proceso de formación relevante y trascendental.

Lo anterior refleja la necesidad de adentrarnos en la comprensión de las estrategias que debemos generar para el desarrollo de competencias en los estudiantes y entender que estos procesos se encuentran articulados a "una práctica de enseñanza consciente de sus limitaciones y debilidades, una práctica que sea capaz de reinventarse y transformarse en la medida en que el que la diseña está también inmerso en un proceso de aprendizaje” (Celis et al., 2020, p. 9).

El diseño de ambientes de aprendizaje influye significativamente en la generación de estrategias didácticas concebidas como detonadoras del desarrollo de las actividades de aprendizaje, pues estas contribuyen y están ligadas a la relación educativa -como segundo criterio de análisis- que se da en el salón de clase; asimismo, propician la construcción de conocimientos, la mejora de las situaciones de aprendizaje y el éxito en las relaciones educativas entre estudiantes y docentes (Rodríguez, 2014).

Las estrategias didácticas involucran a los procedimientos, métodos, técnicas, actividades, relaciones educativas, intracciones pedagógicas, entre otras, que el docente construye y utiliza en su accionar cotidiano en el aula, en correspondencia puede decirse también que son todas aquellas actividades y procesos cognitivos realizados por los estudiantes para el logro de competencias, las cuales "deben ser previamente diseñadas por el maestro, ya que al igual que las estrategias de enseñanza, cada una de las actividades persigue un propósito diferente y, por consiguiente, logran un aprendizaje diferente” (Rodríguez-Garza, Terán-Cázares, Guerra-Rosales y Guerra-Frías, 2016, p. 2750).

Es importante destacar que el trabajo en el desarrollo de competencias considera como estrategia la necesidad de partir de situaciones reales; en ese sentido, el profesor deberá echar mano de su capacidad para construir al interior del aula aquellas situaciones lo más semejantes a la realidad mediante las cuales los estudiantes puedan desarrollar sus competencias.

Entre los aspectos a considerar en la construcción de estrategias didácticas para el desarrollo de competencias se encuentran las particularidades y diferencias individuales de los estudiantes, entendiendo que "Una de las bondades que tiene la educación basada en competencias es que nos permite crear ambientes de aprendizajes planeados de acuerdo a las características de los alumnos (nivel de desarrollo cognitivo, conocimientos previos, factores motivacionales, entre otros)" (Rodríguez, 2014, p. 12). 
Lo anterior implica que los profesores que buscan desarrollar una docencia bajo el enfoque por competencias deberán partir de cambios en su manera de planear y ejercer su práctica docente pues, como se mencionó, sabemos que "hablar de competencias implica un cambio en nuestra planeación docente, una de las principales tareas del docente es la gestión de ambientes de aprendizaje adecuados para que los alumnos participen en un proceso de formación útil y significativo" (Rodríguez, 2014, p. 2).

En suma, puede afirmarse que no es suficiente con el diseño de ambientes de aprendizaje adecuados para el logro de competencias, es menester la construcción y puesta en práctica de estrategias didácticas, y todas aquellas actividades de aprendizaje que se llevan a cabo en el aula para el logro sistemático y organizado de las competencias.

Respecto del segundo criterio -relación educativa- en el momento de la intervención, se aprecia la puesta en común de una docente a sus estudiantes, respecto de lo que va abordar en el tema planeado, la maestra expone la temática con poca réplica por parte de los estudiantes al comparar este evento con una clase "teórica", aquí prevalece el manejo de contenidos prácticos, donde se observa el desarrollo de una temática con fuerte vinculación entre teoría y práctica. La profesora, desde el enfoque por competencias, enfatiza en el manejo de software por parte del estudiante y relaciona su tema con una situación de la vida profesional, destacando sobre posibles situaciones reales que se pueden presentar y la actitud que deba desarrollar el estudiante (Celis et al., 2020).

Si bien es posible diferenciar lo referente a la orientación de las materias teóricas y las teórico-prácticas, en la práctica educativa observada, no se encuentra diferencia alguna. En el grado de exigencia, respecto de la preocupación por concretar un determinado tipo de saberes, se aprecia un mayor énfasis en las materias teóricas, pero en el aula, se da un mayor acercamiento con el grupo de los profesores de materias prácticas. En el periodo observado, los espacios de negociación y el debate de la planeación didáctica, no se apreciaron diferencias en entre las materias teóricas y las prácticas, asumiéndose que la planeación es un lineamiento administrativo impuesto por la institución y que los profesores deben realizar (Rodríguez, 2014).

\section{Intervención en el aula}

Esta categoría aborda el proceso de aprender y enseñar en el aula desde la lógica de la educación basada en competencias. Recoge los resultados de lo analizado en la categoría de planeación didáctica, sobre todo en la intención de mantener los niveles de congruencia que parte de los elementos del concepto de competencia, con lo cual se busca formar de manera diferente, donde los estudiantes logren expresar en desempeños los productos de enfrentar situaciones complejas (Marín et al., 2015).

Conocer el nivel del logro de competencias a partir del trabajo de los contenidos conceptuales, lleva a la reproducción de definiciones, no permite reconocer con fiabilidad si éstas han sido comprendidas y, sobre todo, si el alumno es capaz de aplicarlas en distintos contextos (Zabala y Arnau, 2008).

Los testimonios mostrados en la Tabla 3, dan cuenta de las estrategias implementadas desde la perspectiva de los profesores que abordan materias "teóricas". 
TABLA 3.

Criterios de intervención en el aula en materias teóricas.

\begin{tabular}{|c|c|c|}
\hline \multirow[t]{2}{*}{ Categoría } & Criterios & Testimonios \\
\hline & $\begin{array}{l}\text { Actividades } \\
\text { de apertura }\end{array}$ & $\begin{array}{l}\text { Por equipos vamos a decidir qué es lo más importante. Realizaremos una línea } \\
\text { del tiempo y vamos a decidir qué vamos a poner en ella y vamos a poner lo más } \\
\text { importante de cada época. }\end{array}$ \\
\hline $\begin{array}{l}\text { Intervención } \\
\text { en el aula }\end{array}$ & $\begin{array}{l}\text { Interacción } \\
\text { pedagógica }\end{array}$ & $\begin{array}{l}\text { Les traje un material seleccionado, al finalizar la clase voy a borrar el pizarrón } \\
\text { y no podemos tomar fotografías. En } 10 \text { minutos empezamos a elaborar la línea } \\
\text { del tiempo, si no se acuerdan que es una línea de tiempo les explico que es un } \\
\text { proceso de cómo va evolucionando. La profesora cuestiona a los estudiantes } \\
\text { de qué aportó Roma a la administración, cuando terminan de pasar todos los } \\
\text { equipos, les hace preguntas relacionadas con lo que escribieron, para que ellos } \\
\text { tomen nota, por último, les dice la profesora que terminando de copiar la línea } \\
\text { de tiempo y revisarla pueden salir. }\end{array}$ \\
\hline
\end{tabular}

Fuente: Autores.

En estos testimonios se observa que hay poca diferencia entre las actividades de apertura de una clase tradicional y la basada en competencias. El primer testimonio evidencia rutinas de apego puntual al programa, un abordaje cuya historia es descrita como la suma de hechos que aparecen descontextualizados para el estudiante y la única actividad será memorizar fechas para llenar exámenes de lápiz y papel. También se evidencian prácticas de simulación y poca responsabilidad, en donde se esperaría que el profesor reaccionara ante eventualidades y no que estas se empleen como oportunidad para cancelar o suspender clase. Esto da cuenta también de una forma de señalar al programa y evitar otras miradas necesarias para la interacción con el grupo, por el contrario, testimonios como estos, describen una relación educativa vertical, con pocas posibilidades de comunicación. Se considera la necesidad de "plantear actividades que pueden despertar el interés en los estudiantes y motivarlos a participar y a generar comunidades de aprendizaje que contribuyan a fortalecer y desarrollar habilidades y destrezas en ellos" (Espinosa, González y Hernández, 2019, pp. 110-111).

El segundo testimonio, da cuenta de una interacción pedagógica que encierra un ejercicio autoritario y tradicional, de control y sometimiento hacia el estudiante, muestra una práctica no solo vertical, sino coercitiva para el grupo, donde el profesor habla, interactúa y está frente al pizarrón, escribiendo para un grupo distante, porque la misma visibilidad que tienen del pizarrón es limitada. Una clase "planeada" desde esta perspectiva, evidencia una reproducción lineal de contenidos que el maestro transcribe para luego repetirlos en el pizarrón y borrar rápido, impidiendo que se saquen copias, es decir, el estudiante ha de seguir el ritmo de escritura del profesor. ¿Qué tipo de aprendizaje se esperaría que el estudiante desarrolle ante estas prácticas?, considerando que el estudiante va a la zaga del saber del profesor, el profesor es quien tiene la idea general de lo que pretende comunicar, el estudiante por su parte, está concentrando en copiar del pizarrón, por lo que la posibilidad de integrar la idea completa se va acumulando a las anteriores y posteriores ideas, hasta que terminan por desaparecer. Esto manifiesta que el docente aun enfrenta problemas en cuanto "al manejo del grupo, identificación de las dificultades de los estudiantes, el cierre de la clase, integrar los contenidos conceptuales, 
procedimentales y actitudinales del conocimiento científico y establecer una interacción constante entre docente-estudiante y estudiante-estudiante" (Espinosa, González y Hernández, 2019, p. 111).

También pueden apreciarse en estos testimonios elementos de transición entre una docencia convencional y una por competencias, en donde el docente muestra interés por incorporar la visión y compromiso de los estudiantes para aportar grupalmente, lo que conviene mejor al grupo. Esto da cuenta de posibilidades de delegación de responsabilidades al grupo, por lo que serán estas nuevas tareas las que permitirán que se involucren en sus procesos de formación al ser tomados en cuenta en sus procesos de aprender.

Los testimonios que se describen en la Tabla 4, muestran algunos rasgos de la intervención hecha por profesores que atienden materias prácticas.

TABLA 4.

Citerios de intervención en el aula en materias prácticas.

\begin{tabular}{lll}
\hline Categoría & Criterios & \multicolumn{1}{c}{ Testimonios } \\
\hline & $\begin{array}{l}\text { Actividades } \\
\text { de apertura }\end{array}$ & $\begin{array}{l}\text {.. El profesor les comenta a los estudiantes con qué intención se va a elaborar } \\
\text { importante que ustedes sepan qué van a realizar y también que identifiquen } \\
\text { cuál va ser su actividad concreta en esta. }\end{array}$ \\
$\begin{array}{l}\text { Intervención } \\
\text { en el aula }\end{array}$ & $\begin{array}{l}\text { Miren, vamos a hacer el estado de resultados, con base en este haremos un } \\
\text { flujo efectivo, la forma como la vamos a desarrollar es por medio de porcentajes, } \\
\text { es por ello que haremos primero una parte del ejercicio y enseguida vamos a } \\
\text { proceder para terminarlo. ¿A ver, me han entendido lo que he explicado? ¿Quién } \\
\text { me entendió? ¿Tienen dudas? Pregunta el profesor y todos los alumnos callados } \\
\text { nadie responde, solamente copian lo del pizarrón. }\end{array}$ \\
\hline
\end{tabular}

Fuente: Autores.

Estos testimonios, bajo los mismos criterios de Actividades de apertura e Interacción pedagógica, describen el desempeño de profesores que imparten materias prácticas. Puede observarse cómo los estudiantes se organizan para lograr una mayor comunicación y convivencia entre el grupo para lograr el propósito de la materia. También se evidencia una práctica tradicional de conducción, de consulta concreta para contestar o para anotar un dato faltante, un tanto distante de las estrategias que obstaculizan que el estudiante construya por sí solo, que integre las diferentes voces y aportaciones de sus compañeros de clase. Se contempla que el docente maneja un modelo tradicionalista donde le da todo al estudiante y no lo hace partícipe de lo que se quiere lograr... "yo escribo, explico y ustedes como estudiantes solamente copian"; en algunas ocasiones el estudiante nada más escribe, y no sabe lo que está haciendo, porque al docente lo que le interesa es "revisar el ejercicio en la libreta", pero sin saber en realidad sobre el proceso de construcción del conocimiento, quedando mas como un ejercicico de transmisión de información. Lo anterior muestra que la posición del docente en estas circunstancias debe ser más de apoyo y acompañamiento entendiendo al docente como un facilitador en el aula mediante propuestas concretas "nunca debe proponer una actividad sin brindar una estrategia para su ejecución, en otras palabras, debe anticiparse a la interacción proponiendo dinámicas que posibiliten dicho desarrollo" (Celis et al., 2020, p. 24). 


\section{La evaluación en el enfoque por competencias}

La evaluación en el enfoque por competencias es de sustancial importancia. En la evaluación se hace evidente la diferencia entre un modelo tradicional y un modelo por competencias (Mejía, 2012). Es frecuente apreciar instituciones que han incursionado en nuevos enfoques de hacer una planeación pertinente, pero en la evaluación evidencian que continúan evaluando contenidos y no desempeños como se busca en el enfoque por competencias.

Esta categoría pone especial interés en el criterio desempeños por ser éstos donde se evidencia lo esencial del enfoque por competencias, las prácticas se convierten ahora en los escenarios donde pueden evaluarse los desempeños en los cuales se conjuntan los múltiples saberes o recursos cognitivos. El desempeño, por tanto, es la "evidencia" donde se cristaliza esta suma de recursos que llevan al estudiante enfrentar situaciones complejas. Esta situación lleva a las concepciones de evaluación auténtica como una estrategia importante en la evaluación de competencias, de acuerdo con Villarroel, Bloxham, Bruna, Bruna \& Herrera-Seda (2017) "La autenticidad se entiende como realismo, contextualización y problematización a la hora de enseñar y evaluar contenidos curriculares." (p. 2).

El testimonio que se presenta en la Tabla 5, evidencia un segmento de observación donde se desarrolla la evaluación de una materia teórica.

TABLA 5.

Criterios de la evaluación en materias teóricas

\begin{tabular}{|c|c|c|}
\hline Categoría & Criterios & Testimonios \\
\hline Evaluación & Desempeños & $\begin{array}{l}\text { [...] El profesor repartirá por equipos una etapa de la línea del tiempo y por } \\
\text { equipos ellos decidirán cómo pasan al pizarrón a escribir la línea del tiempo } \\
\text { previamente ya consultada por los estudiantes y el profesor va ir evaluando a } \\
\text { cada equipo, da tiempo para terminar la actividad que se está pidiendo, tomando } \\
\text { asistencia, también les comenta que va evaluar el desempeño en clase y que } \\
\text { todos tienen que terminar y trabajar. Después de un tiempo, el docente les } \\
\text { pregunta si ya terminaron y ellos le dicen, que necesitan más tiempo y les da } \\
\text { tres minutos más para terminar la actividad y empezar a evaluar, por último, } \\
\text { cada uno tiene que escribir la línea del tiempo en su libreta y presentarla para } \\
\text { su evaluación. }\end{array}$ \\
\hline
\end{tabular}

Fuente: Autores.

Se observa cómo el docente, da instrucciones de la manera en que quiere la realización del trabajo y las especificaciones que necesita para su evaluación, con poco margen para destacar el aprendizaje de los estudiantes, así como esa falta de espacio para el estímulo de su autonomía, al monitorear su avance y las interferencias, para comprobar el nivel de comprensión de la lectura que el docente compartió, ello permite identificar las necesidades de cada equipo para elaborar nuevas estrategias, nuevos planteamientos.

Se puede inferir que el docente de la materia teórica le facilita toda la información al estudiante, no lo hace pensar, ellos están esperando que el docente les de las respuestas para ellos copiarlas del pizarrón y tener su punto de evaluación al momento de terminar el ejercicio. 
El objetivo de la evaluación se basa en buscar el grado de enseñanza adquirido de los contenidos de aprendizaje que conforman la competencia de la asignatura, pretendiendo que el estudiante sea capaz de realizar acciones y dar respuestas a los ejercicios vistos en clase; como se puede apreciar, aquí el docente no deja que el estudiante sea autónomo y pueda sacar sus propios errores del ejercicio para su aprendizaje. Esto lleva a la evaluación concebida como una oportunidad para que el estudiante reflexione sobre sus aprendizajes y el docente analice las estrategias empleadas al enfrentar a los estudiantes a situaciones planteadas para el desarrollo de competencias, este proceso "posibilita la metacognición en la medida que el estudiante se ve involucrado en situaciones que en las que debe monitorear conscientemente su proceso y el de los demás" (Celis et al., 2020, p. 19).

Los testimonios que se presentan en la Tabla 6, muestran lo que se hace en una materia práctica.

TABLA 6.

Criterios de la Evaluación en materias prácticas.

\begin{tabular}{|c|c|c|}
\hline Categoría & Criterios & Testimonios \\
\hline Evaluación & Desempeños & $\begin{array}{l}\text { [...] El episodio de la materia teórica, el profesor les comenta a los estudiantes } \\
\text { que se pretende evaluar los estados de resultados, pasa a una estudiante para } \\
\text { que empiece a resolver el ejercicio en el pizarrón y los demás tiene que copiar } \\
\text { para su evaluación, la estudiante tiene que traer calculadora, plumón sino sería } \\
\text { imposible resolver el problema. Los estudiantes solamente copian, pero algunos } \\
\text { no saben lo que están copiando, lo hacen nada más para tener su punto de } \\
\text { evaluación. Por último, el profesor les reparte unas hojas para que resuelvan un } \\
\text { problema de tarea, es lo que deben de entregar mañana, el profesor no recuerda } \\
\text { si hay clases mañana, los alumnos cuestionan al profesor porque tiene que } \\
\text { entregar ese trabajo mañana, no quedó claro la explicación y ya hay que entregar } \\
\text { un producto, el profesor les comenta que tienen que entregar ese ejercicio y seria } \\
\text { su evaluación sin importar si se comprendió o no. }\end{array}$ \\
\hline
\end{tabular}

Fuente: Autores.

Este testimonio ofrece una visión de la exigencia de un desempeño para ser evaluado. Este se presenta como una acción, una oportunidad de llevar a la práctica un ejercicio reflexionado que convierte, a la evaluación y al aula, en el escenario para el diálogo y la interacción, evidencia una evaluación ligada a un hacer reflexivo orientado a la transformación colegiada. Este diálogo propugnan por un cambio analizado a la luz de la teoría y la realidad del aula.

El testimonio refleja una evaluación auténtica, la cual representa hacer un alto en el proceso y reflexionar sobre la forma que se desarrolla para enfrentar y resolver una situación compleja, desde esta lógica, implica que el estudiante sabe desde el inicio, lo que va a presentar como evidencia. Evaluar desde esta perspectiva representa un momento más de aprendizaje (Karim, Abduh, Manda \& Yunus, 2018).

La evaluación de competencias es concebida como una estrategia que debe ser empleada en los procesos de enseñanza y de aprendizaje. Desde esta visión se establecieron, desde la planeación, los criterios de intervención en el aula a fin de promover la reflexión sobre las acciones vividas, considerando que, si bien, una de las facetas que deben ser evaluadas 
corresponde a lo informativo, también se incorpora el aspecto de la reflexión, el cual permite cuestionarse sobre las competencias desarrolladas por los estudinates y el docente, así como los procesos vividos para lograrlo; de esta manera los estudiantes verifican la progresión en el desarrollo de competencias; en síntesis: una evaluación auténtica con rasgos de multireferencialidad, situada y dialógica (Bazmi, Ahmed, Rubena, 2018; Brown, 2015; Celis et al., 2020; Villarroel \& Bruna, 2019).

De manera general, los resultados de la investigación evidenciaron tres grupos de profesores: el primero, refiere a aquellos docentes que han interiorizado los principios de este enfoque con concepciones y práctica congruentes; el segundo, integra a aquellos profesores que han logrado avanzar, que evidencian movimiento en la planeación, aúnque muy poco en la evaluación; y el tercero; está caracterizado por profesores que, por diversas circunstancias, no ha implementado cambios y su práctica transcurre de manera tradicional.

Al respecto, los programas de formación docente en la universidad deben ser fortalecidos a fin de avanzar en el establecimiento y consolidación del modelo por competencias que se pretende implantar en la institución, conscientes de que la transformación más importante radica en los cambios que deban producirse entre los profesores, pues generalmente los grandes cambios pedagógicos son asumidos con mayor celeridad por los estudiantes, encontrándose que son los docentes quienes presentan mayor resistencia al enfoque por competencias, estando siempre latente en los estudiantes la necesidad de que sus profesores adquieran y apliquen el enfoque por competencias en el ejercicio de su profesión aunque esto les resulte una tarea difícil (Sánchez et al., 2014).

\section{CONCLUSiOnes}

Los objetivos del estudio se alcanzaron, pues logró observar, analizar y reflexionar las características de profesores, en cuanto a las estrategias implementadas para concretar en el área de Administración el enfoque por competencias en las tres dimensiones de práctica docente estudiadas. Estas reflexiones fueron fundamentales en las reconstrucciones teóricas hechas en la búsqueda de coherencia entre las interpretaciones conceptuales y la realidad estudiada. Los acuerdos y diálogos en torno a los encuentros y desencuentros dados en la interpretación de los momentos de planeación, intervención y evaluación.

$\mathrm{El}$ análisis documental realizado fue determinante para inferir la manera como fueron reflexionadas las estrategias en el proceso de planeación didáctica de los profesores estudiados. Asimismo, la observación brindó la claridad sobre cómo se dieron las interacciones áulicas, cuáles fueron los fundamentos, creencias y percepciones aplicadas en el aula, relacionadas con el modelo educativo, evidenciando los procesos centrales que caracterizan la práctica educativa en la institución.

Desde el enfoque cualitativo fue posible estudiar a los docentes en un escenario que corresponde con su ambiente natural y cotidiano, observados desde una perspectiva holística, donde el aula de clase y los grupos no fueron reducidos a datos, ni considerados como objetos de estudio, sino como un todo conformado por sujetos en sus momentos de interacción, con un modo natural y no intrusivo. 
Los resultados analizados, muestran pocos cambios en las tres categorías analizadas, más bien, reflejan rastros de prácticas tradicionales. Es necesario intensificar los momentos de capacitación y discusión del modelo por competencias que conduzca a nuevas prácticas educativas, pues el enfoque ha penetrado parcialmente en las aulas. El estudio recoge múltiples interpretaciones sobre los referentes de los profesores, quienes, ante la presión de formar diferente, planear y evaluar distinto, acuden a su experiencia, a la forma cómo ellos fueron formados, limitando así, las posibilidades de incursionar en nuevas prácticas docentes.

Resulta relevante la categorización hecha de los profesores participantes en la investigación, la cual refleja tres grupos de profesores: los convencidos, los que se encuentran en el camino de incorporación y los indecisos. Con esto se concluye que, en la institución investigada, se vive un proceso de transición hacia el enfoque de la educación basada en competencias, y si en lo general la realidad de la práctica, aporta insumos para una transición, es importante incorporar a este proceso la participación de docentes, quienes de una manera más argumentada puedan implementar alternativas de cambio en sus prácticas que permitan un mayor acercamiento y dialogo con sus estudiantes.

Finalmente, es menester entender que el enfoque por competencias implica debate y cambios, desde el analisis del concepto mismo, hasta la necesaria reflexión por parte de los profesores para conocer qué tipo de prácticas serían o no pertinentes y la ocasión para incursionar en alternativas donde otros mundos sean posibles.

\section{REFERENCIAS}

Bazmi, F. D., Ahmed, S. \& Rubena (2018). Impact of using authentic material on the academic achievement of secondary school students. Bodhi: International Journal of Research in Humanities, Arts and Science, 3(S2), 49-77. Available from http://www. bodhijournals.com/pdf/spl/December2018/Bodhi_V3S2.pdf

Brousseau, G. (2007). Iniciación al estudio de la teoría de situaciones didácticas. Buenos Aires: Zorzal.

Brown, R. (2015). La evaluación auténtica: El uso de la evaluación para ayudar a los estudiantes a aprender. Relieve, Revista Electrónica de Investigación y Evaluación. Educativa, 21(2), 1-10. https://doi.org/10.7203/relieve.21.2.7674

Celis, J., Parada, Y. \& Ríos, H. (2020). Acción, evaluación y reflexión. De la contemplación a la expresión: una ruta del pensamiento. Praxis Pedagógica, 20(26), 6-32. https:// doi.org/10.26620/uniminuto.praxis.20.26.2020.6-32

Robles-Haros, B. I. y Estévez-Nenninger, E. H. (2016). Enfoque por competencias: problemáticas didácticas que enfrentan el profesorado. Educare, 20(1), 1-12. http://dx.doi. org/10.15359/ree.20-1.25

Chavarría-Zambrano, P. I. y Camacho, H. (2018). Ruta metodológica en la investigación etnográfica. Polo del Conocimiento. 3(12), 449-468. https://doi.org/10.23857/ pc.v3i12 
Cifuentes, J., Cortés, L., Garzón, N. y Gozález, D. (2020). Desarrollo de las competencias de indagación y explicación a través de prácticas de aula basadas en la enseñanza para la comprensión. Cultura Educación y Sociedad, 11(2), 87-109. https://doi. org/10.17981/cultedusoc.11.2.2020.06

Domingo, Á. (2021). La Práctica Reflexiva: un modelo transformador de la praxis docente. Zona próxima, (34), 1-21. https://doi.org/10.14482/zp.34.370.71

Eberle, T. (2019). Análisis fenomenológico del mundo de la vida e investigación social interpretativa: La importancia del postulado de adecuación en la obra de Alfred Schutz. Revista Diferencias, 4(7), 137-153. Recuperado de http://www.revista.diferencias.com.ar/index.php/diferencias/article/view/171

Espinosa, E., González, K. y Hernández, L. (2019). Implicaciones de la reflexión y la mediación didáctica en docentes en formación durante su práctica educativa. Revista Científica, 34(1), 101-122. https://doi.org/10.14483/23448350.13442

Guzmán, I., Marín, R. e Inciarte, A. (2014). Innovar para Transformar la Docencia Universitaria. Maracaibo: Universidad de Zulia.

Guzmán, I. y Marín, R. (2011). La Competencia y las Competencias Docentes: reflexiones sobre el Concepto y la Evaluación. Revista Electrónica Interuniversitaria de Formación del Profesorado, 14(1), 151-163. Disponible en https://revistas.um.es/reifop/ issue/view/11941

Jiménez-Domínguez, B. (2000). Investigación cualitativa y psicología social crítica. Contra la lógica binaria y la ilusión de la pureza. Revista Universidad de Guadalajara, (17). Disponible en http://www.cge.udg.mx/revistaudg/rug17/3invesigacion.html (consultada en 2000)

Karim, A. A., Abduh, A., Manda, D. \& Yunus, M. (2018). The effectivity of authentic assessment-based character education evaluation model. TEM Journal, 7(3), 495-500. https://doi.org/10.18421/TEM73-04

Londoño, D. y Luján, D. (2020). Competencias científicas en docentes de la ciudad de Medellín, Colombia: Análisis desde la formación docente. Cultura, Educación y Sociedad, 11(1). 39-54. http://dx.doi.org/10.17981/cultedusoc.11.1.2020.03

Marín, R., Guzmán, I., Inciarte, A. y Araya, E. (2015). Intervenir e investigar en el aula. Experiencias en la formación de profesores. Buenos Aires: Alfagrama.

Mejía, O. (2012). De la Evaluación Tradicional a una Nueva Evaluación Basada en Competencias. Revista Electrónica Educare, 16(1), 42-58. https://doi.org/10.15359/ree.161.3

Morales, R. (2018). La planeación de la enseñanza-aprendizaje, competencia que fortalece el perfil docente. Revista Iberoamericana para la Investigación y el Desarrollo Educativo, 8(16), 1-24. https://doi.org/10.23913/ride.v8i16.343 
Moria, E., Refnaldi, R. \& Zaim, M. (2017). Using Authentic Assessment to Better Facilitate Teaching and Learning: The Case for Students' Writing Assessment. Advances in Social Science, Education and Humanities Research (ASSEHR), 148, 333-337. https://doi.org/10.2991/icla-17.2018.57

Palma, K. (2017). Los principios didácticos constructivistas como prácticas inclusivas en el aula de primaria. Innovaciones educativas, 19(27), 41-54. https://doi.org/10.22458/ ie.v19i27.1954

Penagos, G. (2015). Desde los proyectos de aula hacia la investigación formativa: un reto de la docencia en la educación superior. Rastros Rostros, 17(31), 105-109. Disponible en https://revistas.ucc.edu.co/index.php/ra/article/view/745

Rodríguez, H. (2016). Importancia de la formación de los docentes en las instituciones educativas. Ciencia Huasteca, boletín científico de la Escuela Superior de Huejutla. Estado de Hidalgo, 5(9). https://doi.org/10.29057/esh.v5i9.2219

Rodríguez, H. (2014). Ambientes de Aprendizaje. Ciencia Huasteca, 4(7). Disponible en https://www.uaeh.edu.mx/scige/boletin/huejutla/n4/e1.html

Rodríguez-Garza, B., Terán-Cázares, M., Guerra-Rosales, A. y Guerra-Frías, M. (2016). Orientaciones básicas en el diseño de estrategias didácticas para la construcción de aprendizajes en los nuevos escenarios educativos. Vinculatégica EFAN, 2(1), 2892-2913. Recuperado de http://www.web.facpya.uanl.mx/vinculategica/Revistas/R2/2744-2765\%20-\%20Orientaciones\%20Basicas\%20En\%20El\%20Diseno\%20 De\%20Estrategias\%20Didacticas\%20Para\%20La\%20Construccion\%20De\%20 Aprendizajes\%20En\%20Los\%20Nuevos\%20Escenarios\%20Educativos.pdf\#page=1

Roegiers, X. (2016). Marco conceptual para la evaluación de las competencias. [IBE/2016/ $W P / C D / 04]$. Ginebra: Oficina Internacional de Educación de la UNESCO (OIEUNESCO). Disponible en http://www.ibe.unesco.org/es/documento/marco-conceptual-para-la-evaluaci\%C3\%B3n-de-competencias

Roegiers, X. (2010). Pedagogía de la Integración. Competencia e Integración de los conocimientos en la enseñanza. México, D.F.: FCE.

Sánchez, F., Soler, A.. López, D.. Martín, C.. Ageno, A., Belanche, L., Cabré, J., Cobo, E., Farré, R., García J. \& Marés P. (oct. 2014). Developing Professional Skills at Tertiary Level: A Model to Integrate Competencies across the Curriculum. IEEE Frontiers in Education Conference Proceedings, FIE, Madrid, Spain, pp. 1-9. http:// doi.org/10.1109/FIE.2014.7044166

Soto, A. y Nieves, M. (2018). Enseñar y aprender a investigar etnografía en educación. Plural. Antropologías desde américa latina y el caribe. 1(1). 161-186. Disponible en https://asociacionlatinoamericanadeantropologia.net/revistas/index.php/plural/article/view/22

UPCH. (2009). Universidad Politécnica de Chihuahua. [Online]. Disponible en http:// www.upchihuahua.edu.mx 
Vargas, C. I. y Romero. G. R. (junio, 2018). Aprendizaje por resolución de problemas en la cátedra de Administración Gerencial de la carrera de Ingeniería en Sistemas de Información de la Facultad Regional Resistencia de la Universidad Tecnológica Nacional. Conferencia presentada al XIII Congreso de Tecnología en Educación y Educación en Tecnología, RedUNCI, Posadas, Argentina. Disponible en http://sedici.unlp.edu.ar/ handle/10915/69080

Villarroel, V. y Bruna, D. (2019). ¿Evaluamos lo que realmente importa? el desafío de la evaluación auténtica en educación superior. Revista Calidad en la Educación, 50, 492-509. http://dx.doi.org/10.31619/caledu.n50.729

Villarroel, V., Bloxham, S., Bruna, D., Bruna, C. \& Herrera-Seda, C. (2017). Authentic assessment: Creating a blueprint for course design. Assessment \& Evaluation in Higher Education, 43(5), 840-854. https://doi.org/10.1080/02602938.2017.1412396

Zabala, A. y Arnau, L. (2008). 11 ideas claves: como aprender y enseñar competencias. Barcelona: GRAÒ.

Isabel Guzmán Ibarra. Profesora-investigadora de la Facultad de Filosofía y Letras de la Universidad Autónoma de Chihuahua (México). Doctora en Ciencias de la Educación. Responsable del Cuerpo Académico Consolidado "Educación y Comunicación”, integrante de varias Redes de investigación. Línea de investigación: formación de profesores, donde ha desarrollado varias investigaciones. Ha dirigido tesis doctorales. Autora de libros, artículos y capítulos de libros sobre el tema de formación y evaluación de profesores. Profesora invitada por universidades nacionales e internacionales, impartiendo seminarios doctorales. Directora y asesora en diseños curriculares de licenciatura y posgrado en universidades nacionales. Estancias realizadas en Universidades de Alcalá de Henares, (Girona), Oberta de Cataluña (Cataluña), Autónoma de Barcelona (Barcelona), de Zaragoza (Zaragoza), España; Ca' Foscari (Venecia) en Italia; Lovaina, en Bélgica; Zulia, en Venezuela; Santiago de Cali (Cali), Simón Bolívar (Barranquilla) e Industrial de Santander (Bucaramanga), en Colombia. https://orcid.org/0000-0001-6075-5024

Rigoberto Marín Uribe. Profesor investigador de la Facultad de Ciencias de la Cultura Física de la Universidad Autónoma de Chihuahua (México). Doctor en Ciencias de la Educación; y miembro del Cuerpo Académico Consolidado "Educación y Comunicación", integrante de varias Redes de investigación. Su línea de investigación formación de profesores, ha dirigido tesis doctorales. Autor de libros, artículos y capítulos de libros sobre el tema de formación y evaluación de profesores y desarrollado varios proyectos de investigación. Profesor invitado por universidades nacionales e internacionales, impartiendo seminarios doctorales y participante en proceso de diseños curriculares. Ha realizado estancias en Universidades de Alcalá de Henares (Girona), Universidad Oberta (Cataluña), Universidad Autónoma de Barcelona (Barcelona), y de Zaragoza (Zaragoza), en España; Universidad Ca'Foscari (Venecia), en Italia; Universidad de Lovaina, en Bélgica; Universidad de Zulia, en Venezuela; Universidad de Santiago de Cali (Cali), Universidad Simón Bolívar (Barranquilla), y la Universidad Industrial de Santander (Bucaramanga), en Colombia. Dirige y asesora diseños curriculares a nivel de licenciatura y posgrado en varias universidades del país. https://orcid.org/0000-0002-8672-4047 
Cintia Lizzette Ortega Alderete. Egresada de la Maestría de Innovación Educativa, actualmente se desempeña como Profesor de Asignatura en la Universidad Politécnica de Chihuahua (México), con una antigüedad de 13 años como docente, con una formación académica de Ingeniería Industrial, especialidad en Calidad y Producción teniendo cursos y certificaciones en el ámbito industrial y académico. https://orcid.org/0000-0002-7501-674x 